\title{
Diagnostic Value of Fine Needle Aspiration BRAFV600E Mutation Analysis in Papillary Thyroid Cancer: A systematic review and meta-analysis
}

\author{
Naif Fnais, Charlene Soobiah, Khalid AL-Qahtani, Jemila S. \\ Hamid, Laure Perrier, Sharon E. Straus, Andrea C. Tricco
}

Version Post-print/Accepted Manuscript

Citation Fnais N, Soobiah C, AL-Qahtani K, Hamid JS, Perrier L, Straus SE, (published version) Tricco AC. Diagnostic value of fine needle aspiration-BRAFV600E mutation analysis in papillary thyroid cancer: A systematic review and meta-analysis. Hum Pathol. 2015 Oct;46(10):1443-54.

Copyright/License

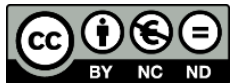
This work is licensed under the Creative Commons Attribution-NonCommercial-NoDerivatives 4.0 International License. To view a copy of this license, visit http://creativecommons.org/licenses/by-nc-nd/4.0/.

\section{How to cite TSpace items}

Always cite the published version, so the author(s) will receive recognition through services that track citation counts, e.g. Scopus. If you need to cite the page number of the author manuscript from TSpace because you cannot access the published version, then cite the TSpace version in addition to the published version using the permanent URI (handle) found on the record page.

This article was made openly accessible by $U$ of $T$ Faculty. Please tell us how this access benefits you. Your story matters. 


\section{Accepted Manuscript}

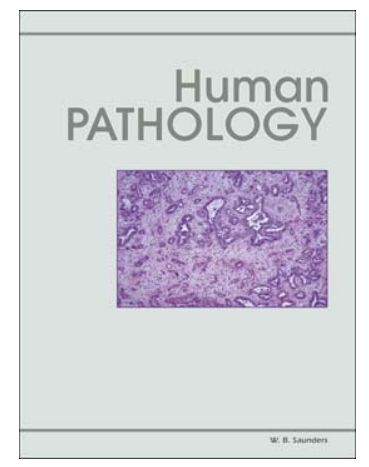

Diagnostic Value of Fine Needle Aspiration $B R A F^{V 600 E}$ Mutation Analysis in Papillary Thyroid Cancer: A systematic review and meta-analysis

Naif Fnais MD, Charlene Soobiah HBSc, Khalid AL-Qahtani MD, MSc, FRCSc, Jemila S. Hamid PhD, MSc, Laure Perrier MLIS, Sharon E. Straus MD, MSc, Andrea C. Tricco PhD, MSc

PII: $\quad$ S0046-8177(15)00206-3

DOI: $\quad$ doi: $10.1016 /$ j.humpath.2015.06.001

Reference: $\quad$ YHUPA 3609

To appear in: $\quad$ Human Pathology

Received date: $\quad 30$ September 2014

Revised date: $\quad 20$ May 2015

Accepted date: 3 June 2015

Please cite this article as: Fnais Naif, Soobiah Charlene, AL-Qahtani Khalid, Hamid Jemila S., Perrier Laure, Straus Sharon E., Tricco Andrea C., Diagnostic Value of Fine Needle Aspiration $B R A F^{V 600 E}$ Mutation Analysis in Papillary Thyroid Cancer: A systematic review and meta-analysis, Human Pathology (2015), doi: 10.1016/j.humpath.2015.06.001

This is a PDF file of an unedited manuscript that has been accepted for publication. As a service to our customers we are providing this early version of the manuscript. The manuscript will undergo copyediting, typesetting, and review of the resulting proof before it is published in its final form. Please note that during the production process errors may be discovered which could affect the content, and all legal disclaimers that apply to the journal pertain. 


\section{Diagnostic Value of Fine Needle Aspiration $B R A F^{V 600 E}$ Mutation Analysis in Papillary Thyroid Cancer: A systematic review and meta-analysis}

Naif Fnais, MD; ${ }^{1}$ Charlene Soobiah, HBSc $;{ }^{2}$ Khalid AL-Qahtani, MD, MSc, FRCSc $;{ }^{1}$ Jemila S. Hamid, $\mathrm{PhD}, \mathrm{MSc} ;{ }^{2,3}$ Laure Perrier, MLIS; ${ }^{2}$ Sharon E. Straus, MD, MSc; ${ }^{2,5}$ Andrea C. Tricco, PhD, MSc. ${ }^{2,6}$

1. Department of Otolaryngology-Head and Neck Surgery, College of Medicine, King Saud University, Riyadh, Saudi Arabia;

2. Li Ka Shing Knowledge Institute of St Michael's Hospital, Toronto, Ontario, Canada;

3. Department of Clinical Epidemiology \& Biostatistics, Faculty of Health Sciences, McMaster University, Hamilton, Ontario, Canada;

5. Division of Geriatrics, Faculty of Medicine, University of Toronto, Toronto, Ontario, M5S 1A8, Canada;

6. Division of Epidemiology, Dalla Lana School of Public Health, University of Toronto, Toronto, Ontario, M5S 1A8, Canada.

\section{Corresponding Author:}

Andrea C. Tricco; MSc, $\mathrm{PhD}$

Scientist, Li Ka Shing Knowledge Institute of St. Michael's Hospital

38 Shuter Street, Room 721, Toronto, ON M5B 1T8

Email: triccoa@smh.ca

Telephone: 416-864-6060 ext 77521/ Fascimile: 416-864-6057

Running title: Diagnostic Value of $B R A F^{V 600 E}$ mutation analysis

Word count: 226 (abstract), 2415 (main text).

Key words: Papillary thyroid cancer; $\mathrm{BRAF}^{\mathrm{V} 600 \mathrm{E}}$ mutation; systematic review; meta-analysis 


\section{Abstract}

Fine needle aspiration (FNA) with cytological analysis is an initial step in diagnosing thyroid nodules that are suspicious for cancer. We systematically reviewed the test accuracy of Btype Raf kinase $\left(B R A F^{V 600 E}\right)$ gene mutation analysis plus conventional FNA in the diagnosis of papillary thyroid cancer (PTC). We identified studies reporting $B R A F^{V 600 E}$ mutation analysis following FNA for evaluation of thyroid nodules through searching MEDLINE, EMBASE, and the Cochrane Central Register of Controlled Trials, scanning reference lists of relevant studies, and contacting experts. Two independent reviewers screened literature results, abstracted data and appraised study quality. When appropriate, bivariate and univariate random effects meta-analyses of sensitivity and specificity were considered for all outcomes. Forty-seven studies met our inclusion criteria after screening 1560 citations and 169 full-text articles. The included studies enrolled approximately 16,170 patients with 9,924 fine needle aspiration samples evaluated for $B R A F^{V 600 E}$ mutation. Univariate-pooled sensitivity was $69 \%(95 \% \mathrm{CI} ; 61 \%, 76 \%)$ for papillary thyroid cancer. For thyroid nodules that were diagnosed cytologically as suspicious for papillary thyroid cancer, univariatepooled sensitivity using fine needle aspiration and $B R A F^{V 600 E}$ results was $52 \%(95 \% \mathrm{CI}$; $39 \%, 64 \%$ ). Despite its high specificity, our meta-analysis shows that $B R A F^{V 600 E}$ mutation analysis has a low sensitivity in diagnosing papillary thyroid cancer in thyroid nodules. The feasibility of this test as a single molecular tool is not well established, which indicates the need for well designed prospective clinical studies. 


\section{Introduction}

Papillary thyroid cancer (PTC) represents the most common histologic type of thyroid carcinomas, accounting for approximately $85 \%$ of thyroid cancers [2,3]. PTC ranks as the eighth most common malignancy diagnosed in women and the 5-year survival rate in the US is approximately $95 \%$ [4-6].

An active mutation in the gene for the B-type Raf kinase (BRAF) has been recently described and represents the most common genetic alteration in PTC [6]. It involves a missense mutation resulting in the substitution of thymine $(T)$ for adenine $(A)$ at nucleotide 1799. Subsequently, valine is replaced with glutamate at residue 600 , which has been found exclusively in PTC (known as $B R A F^{V 600 E}$ ) [8,9]. BRAF is a key component in cell proliferation, differentiation and apoptosis. Thus, $B R A F^{V 600 E}$ mutation is associated with thyroid cancer through up regulation of cell division and proliferation leading to tumor genesis $[10,11]$. Furthermore, $B R A F^{V 600 E}$ mutation was shown to be associated with extrathyroid extension, lymph node metastasis, distant metastasis, advanced tumor-nodemetastasis (TNM) stages, and cancer recurrence, which are considered to be poor prognostic factors [12-16]. Fine needle aspiration (FNA) biopsy with cytological analysis is an initial step in the diagnosis of thyroid nodules and pathology $[17,18]$.

According to the "Bethesda System for Reporting Thyroid Cytopathology", there are 6 general diagnostic categories for FNA cytology: I. nondiagnostic or unsatisfactory, II.benign, III. atypia of undetermined significance, IV. follicular neoplasm or suspicious for a follicular neoplasm, V. suspicious for malignancy, and VI. malignant [19]. FNA findings interpreted as categories III-V (often reported as "indeterminate”) occur in about 10 to $30 \%$ of FNA results and are considered a significant limitation in the investigation of thyroid nodules $[17,20]$, making the intraoperative frozen section analysis a means of guiding for further planning $[21,22]$. If the accuracy of FNA can be improved by adding $B R A F^{V 600 E}$ mutation analysis to 
the conventional FNA, this may help with the investigation of these nodules.

We aimed to systematically review the test accuracy of the cytologic determination of $B R A F^{V 600 E}$ mutational status plus conventional FNA in patients with thyroid nodules suspicious for cancer. 


\section{Materials and Methods}

A systematic review protocol based on the PRISMA Statement for Protocols was used to guide the reporting of this review [23]. A draft protocol was assembled, circulated to systematic review methodologists and clinicians, and revised as necessary. It is available upon request from the authors upon request.

\section{Eligibility criteria}

Studies eligible for inclusion were diagnostic randomized trials, cohort, and case control studies that were conducted in a clinical setting, reported on test accuracy, and written in English and Arabic, as these were the fluent languages of the authors. Patients were those aged 16 years or older who had thyroid nodules. The index test was FNA and $B R A F^{V 600 E}$ mutation analysis. $F N A-B R A F^{V 600 E}$ samples were not considered if they came from histological tissue, paraffin-embedded, frozen or microdissected samples. Our outcome of interest was confirmed PTC, obtained through histological assessment of tissue obtained from biopsy, surgery or autopsy (i.e. reference test).

\section{Search}

An experienced information specialist (LP) conducted all of the literature searches in consultation with the research team. The search strategy for the main electronic search (MEDLINE) is presented in the eFile 1. It was modified as necessary for EMBASE and the Cochrane Central Register of Controlled Trials and is available from the authors upon request. Medical Subject Headings and text words related to FNA and thyroid cancer were used to search MEDLINE (OVID Interface) and EMBASE (OVID Interface) from inception until November 18, 2014. The search was not limited by study design or date of dissemination. Additional articles were 
identified through scanning the reference lists of included studies and screening conference symposia abstracts.

\section{Study selection}

A pilot-test was conducted prior to embarking on screening, which only occurred with high inter-rater agreement was achieved across the team. Subsequently, two reviewers independently screened all identified titles and abstracts for inclusion using a pre-defined inclusion criteria form. The full-text of potentially relevant articles were obtained and assessed in the same manner. Discrepancies were resolved by discussion or the involvement of a third reviewer.

\section{Data collection process}

A draft data extraction form was developed, piloted, and modified, as necessary. Two reviewers extracted all of the data using the standardized data extraction form, independently. Discrepancies were resolved by discussion or the involvement of a third reviewer. The data were checked a third time to ensure data integrity. When multiple study publications reported data from the same population (i.e. a companion report), the study reporting the largest sample size was considered the major publication and the other report(s) was used for supplementary data only.

\section{Data items}

The extracted data included study characteristics (e.g., study design, sample size, method of enrollment, setting, method of $B R A F^{V 600 E}$ mutation assessment, cytological and histologic characteristics of the samples were obtained), patient characteristics (e.g., mean age, gender), and outcome results (e.g., number of true 
positive, true negative, false positive, and false negative samples required to compile a $2 \times 2$ table for the sensitivity and specificity for each study).

\section{Methodological quality}

The methodological quality of the included studies was assessed using the Cochrane Handbook for Systematic Reviews of Diagnostic Test Accuracy Tool, Version 1.0.0. This 20-item tool assesses the following domains: representative spectrum, acceptable reference standard, acceptable delay between tests, partial verification avoided, differential verification avoided, relevant clinical information, uninterpretable results reported, withdrawals explained, unchanged technology of the index test, clear definition of what was considered to be a "positive" result, appropriate training of test operators, treatment withheld until both the index test and the reference standard were performed, data on observer variation reported and within an acceptable range, data on instrument variation reported and within an acceptable range, pre-specification of objectives, and free from commercial funding [24]. The "established cut-off values" criterion was excluded from our assessment because none of the studies used these.

\section{Synthesis of results}

The Bethesda classification scheme was used to categorize the findings of the included studies [19]. Subsequently, sensitivity of $F N A-B R A F^{V 600 E}$ mutation were calculated for the included studies and presented in forest plots. Bivariate metaanalysis of sensitivity and specificity was considered for all outcomes and summary receiver operating characteristic (sROC) curves were estimated, when appropriate. In situations where, specificity was either not defined (false positive and true negative values are zero) or was reported as $100 \%$ (perfect specificity) for all the studies, a univariate random effects meta-analyses were performed to provide pooled estimates 
of sensitivity and their corresponding $95 \%$ confidence intervals. Statistical heterogeneity among the studies was investigated using the $\mathrm{I}^{2}$ statistic $[25,26]$. All analyses were performed using the R statistical package [27]. 


\section{Results}

\section{Study selection}

The literature search yielded 1560 citations (i.e., titles and abstracts; Figure 1). In total, 169 full-text articles were retrieved and examined for relevance. A total of 47 studies fulfilled the inclusion criteria and were included [6,12,20-22,28-69].

\section{Study and patient characteristics}

Around $50 \%$ of the included studies were case control studies $(n=26 / 47)$, and the remaining were prospective cohort studies. 14 studies were conducted in the USA $[6,12,21,31,32,42,44,45,54,56-58,65,67], 15$ studies in Korea [20,22,36-39,41,49$51,59,60,62,63,68], 12$ in Italy $[29,33-35,40,43,46,47,55,61,64,66]$, two in China [48,52], and one study each were from Japan [28], Portugal [30] Germany [53] or the UK [69]. All studies were conducted between 2004 and 2014 and the number of patients ranged from 10 to 6,402 with a mean age of 47.5 (Tables 1 and 2).

\section{Methodological quality assessment results}

At least $70 \%$ (>29/47) of the included studies adequately addressed 12 of the quality assessment items (Supplementary Table). In contrast, only one study clearly reported an acceptable delay between tests, 3 studies conducted the reference standard without knowledge of the index test (this was scored as no in 30 studies and unclear in the rest), 3 studies conducted the index test without knowledge of the reference standard (this was scored as no in 27 studies and unclear in the rest), 12 studies described withdrawals (this was scored as no in 25 and unclear in the rest), 4 studies withheld treatment until the reference standard/index test were assessed (this was scored as no in 20 studies and unclear in the rest), 4 studies reported data within an acceptable range for observer variation (this was scored as no in the rest of the 
studies), and 46 of the studies did not report data on instrument variation within an acceptable range (this was scored as unclear in one study).

\section{Meta-analysis results}

Indeterminate group (Bethesda categories III-V)

Seven studies reported this outcome. Among them, 1 reported zero false positive and true negative values, and hence specificity could not be estimated [32]. For the remaining 6 studies, specificity is reported as $100 \%$, consequently pooling the estimates was not necessary. We performed a univariate random effects meta-analysis to pool sensitivity of $B R A F^{V 600 E}$ mutation across the 7 studies that reported the outcome. The pooled sensitivity estimate was $30 \%$ (95\% confidence interval [CI]; $11 \%, 50 \%),\left(\mathrm{I}^{2}=79.54 \%\right)$. The forest plot for sensitivity is presented in figures 2 . Atypia of undetermined significance

Four studies reported this outcome, among them specificity and sensitivity could not be estimated for 3 studies due to data ambiguity. We, therefore, performed a univariate random effects meta-analysis to pool sensitivity estimates from one study that examined $B R A F^{V 600 E}$ mutation among the atypia of undetermined significance group as diagnosed by the reference standard [44]. The pooled sensitivity was $31 \%$, $(95 \% \mathrm{CI} ; 6 \%, 56 \%)$.

\section{Suspicious for malignancy}

Nine studies reported this outcome. Among them, 4 reported zero false positive and true negative values, and hence specificity could not be estimated $[40,44,55,58]$. For the remaining 5 studies, specificity is reported as $100 \%$, consequently pooling the estimates was not necessary. We performed a univariate 
random effects meta-analysis to pool sensitivity of $B R A F^{V 600 E}$ mutation across the nine studies that reported the outcome. The pooled sensitivity estimate was 52\% (95\% CI; 39\%, 64\% $),\left(\mathrm{I}^{2}=77.42 \%\right)$. The forest plot of sensitivity is presented in figure 3 .

\section{Malignancy - PTC}

Twenty-seven studies reported this outcome in which 19 studies showed high heterogeneity. Specificity can only be estimated for one study [50], since both true negative and false positive values are zero for all the remaining studies, consequently, specificity could not be estimated (Figure 4). For Kwak et al 2010, there were only 3 true negatives and all of them were detected indicating $100 \%$ specificity [50]. Therefore, we performed a univarite random effect meta-analysis to pool sensitivity estimates across the 19 studies. The pooled sensitivity estimate of $B R A F^{V 600 E}$ mutation in identifying patients with malignant PTC was 69\% (95\% CI; 61\%, $76 \%),\left(\mathrm{I}^{2}=90.37 \%\right)$. The forest plot of sensitivity is presented in figure 4 .

\section{RET/PTC rearrangement}

We further extracted data from the included studies related to another common mutation in PTC (RET/PTC rearrangement) for comparison purposes. Only six studies examined this mutation along with $B R A F^{V 600 E}$ mutation $[29,30,33,35,46,53]$. The coupled forest plot for sensitivity/specificity and the sROC curve are presented in figures $5 \mathrm{a}$ and $5 \mathrm{~b}$, respectively. 


\section{Discussion}

Our meta-analysis shows that $B R A F^{V 600 E}$ mutation analysis of FNA cytology has only $30 \%$ sensitivity for PTC in those cases that were reported as indeterminate. Similarly, among suspicious for malignancy FNA cytology group, $B R A F^{V 600 E}$ mutation analysis has a sensitivity of $52 \%$. Moreover, the cytological determination of $B R A F^{V 600 E}$ mutational status in PTC cytology group was correlated with the final pathologic diagnosis with only $69 \%$ sensitivity. Our collective results suggest that the proportion of patients with PTC that are correctly identified using $F N A-B R A F^{V 600 E}$ is low, although specificity overall is shown to be very high. These results are consistent with similar previous meta-analysis results by Jia et al [70]. However, our review is more comprehensive with 8 times the number of included studies and provides an upto-date assessment of this issue. Moreover, we stratified our findings according to Bethesda System for Reporting Thyroid Cytopathology to give a more accurate estimate on the test sensitivity and specificity among different cytologic findings [70].

Several studies have shown that $B R A F^{V 600 E}$ mutation is an indicator of poor disease prognosis since it's associated with advanced disease stage, and cancer recurrence [71]. More recently, the presence of $B R A F^{V 600 E}$ mutation was significantly associated with increased cancer-related mortality among patients with PTC [72].

Therefore, early recognition and preoperative determination of $B R A F^{V 600 E}$ mutation is thought to be of great value when determining the management and operative strategy. For example, recent studies suggest a prophylactic central compartment lymph node dissection done simultaneously with total thyroidectomy for patients with positive $B R A F^{V 600 E}$ mutation $[73,74]$. However, our data shows that $B R A F^{V 600 E}$ screening is unlikely to alter the initial management strategy considering the significantly low sensitivity of this test which cannot consistently rule out PTC when 
tests are negative. Therefore, the use of $B R A F^{V 600 E}$ as a single molecular test is not clinically practical. Moreover, our data confirms an important limitation highlighted by Xing et al, in which $B R A F^{V 600 E}$ mutation will be of limited value with non-PTC samples, since it is mostly associated with PTC [75].

Since it was first introduced, FNA cytology provided great advantages over surgical evaluation and true cut biopsy. It provided simple and quick method for evaluating thyroid nodules, and was well tolerated pain by most patients. However, like any other diagnostic technique, FNA is not without its disadvantages. For example, FNA findings interpreted as non-diagnostic in about 10 to $30 \%$ of FNA results. This important limitation led researchers to work on more accurate and less invasive way to diagnose suspicious thyroid nodules including the $B R A F^{V 600 E}$ mutational analysis, which is generally (as per our meta-analysis) insufficient when it is done alone for diagnostic purpose. Moreover, additional markers have been examined to improve the sensitivity of this test. For example, miRInformTM (Asuragen), which is a panel that consists of seven validated molecular markers “mutations" (KRAS, BRAF, HRAS, NRAS, RET/PTC 1, RET/PTC3 and PAX8/PPAR $)$ [76]. As per our results, very few of the included studies accounted for other mutations (e.g. only 6 studies reported the RET/PTC rearrangement) $[28,29,32,34,45,52]$. Thus, we cannot comment on the utility of combining $B R A F^{V 600 E}$ mutation with other mutations. However, upon further analysis, RET/PTC rearrangement showed slightly higher sensitivity as compared to $B R A F^{V 600 E}$ mutation $[29,30,33,35,46,53]$. Furthermore, the clinical applications of these panels have not been established. Therefore, implementing these panels with different mutations may not be feasible when individual variations between institutions and endocrine pathologists are considered. 
The revised American Thyroid Association (ATA) management guidelines for patients with thyroid nodules and differentiated thyroid cancer strongly recommends doing a lobectomy or total thyroidectomy for suspicious/indeterminate thyroid nodules depending on the lesion's size and other risk factors [17]. According to the ATA, the use of molecular markers including $B R A F^{V 600 E}$ might be considered depending on the clinico-pathological information and physician's judgment [77].

The large number of included studies and patients analyzed with worldwide geographic variation represents a major strength of our systematic review. However, there are some limitations. More than $50 \%$ of the included studies were conducted in three countries (USA, Italy, Korea). Therefore, our conclusion may not be generalizable to all patients with thyroid nodules globally. Moreover, data were pooled across studies from different institutions and countries where variation in the technical accuracy may alter the overall sensitivity and specificity. However, most studies used the same tests to examine the presence of $B R A F^{V 600 E}$ mutation (e.g. PCR, DNA sequencing). All studies were conducted recently and none of our analyses showed any statistical heterogeneity.

The methodological quality of the included studies was comparable. The majority of the included studies used a sampling strategy that is likely representative of the patients who will receive the test in practice. All included studies used acceptable reference standard, which will likely classify the target condition correctly, and reported relevant clinical information, where the same clinical data available when test results were interpreted as would be available when the test is used in practice. The fact that reference standard results were interpreted without knowledge of the results of the index test in only 3 studies, and the index test results were interpreted without knowledge of the results of the reference standard results in 4 
studies are an important limitation worth mentioning. Failure to assess the index and reference test results blindly can lead to more optimistic conclusions. This limitation can only be avoided by using blind assessment of the results in well-designed prospective clinical studies and ideally randomized clinical trials. Moreover, half of the included studies were case control studies which can falsely overestimate the accuracy of a diagnostic test [78]. Finally, many of the studies did not adequately report the results in a clear manner or were ambiguous; as such, we were unable to include these in our analysis.

In summary, we found no strong evidence to support the implementation of $B R A F^{V 600 E}$ mutation analysis plus FNA as a single screening test for patients with suspicious and indeterminate thyroid nodules. This conclusion was exclusively based on the literature, which may differ from clinical practice. Preoperative risk stratification should be considered the main guidance for the best management plan in individual patients. Other diagnostic tests should be the focus of further clinical studies.

\section{Acknowledgements}

We wish to thank Drs. Muhammad Mamdani, Anna Sawka and Hend Aldrees for their assistance and guidance in the planning stages of this review and Dr. Rabab Alshahrani for helping with screening and abstracting data.

Author Contributions: Drs. Fnais and Tricco had full access to all of the data in the study and take responsibility for the integrity of the data and the accuracy of the data analysis.

Study concept and design: Drs. Fnais, Tricco and Straus. 
Acquisition of data: Drs Fnais and Tricco, and Ms. Soobiah and Perrier.

Analysis and interpretation of data: Drs Fnais and Tricco, and Ms. Soobiah.

Drafting of the manuscript: Drs Fnais, Tricco and Al-Qahtani, and Ms. Soobiah.

Critical revision of the manuscript for important intellectual content: Drs Fnais,

Tricco, Straus, Hamid and Al-Qahtani, and Ms. Soobiah.

Statistical analysis: Dr. Jemila S. Hamid.

Administrative, technical, or material support: Drs. Fnais and Tricco, and Ms.

Perrier.

Study supervision: Drs Fnais, Tricco and Straus.

Funding/Support: This review was conducted as part of a systematic review course taught by Drs. Straus and Tricco through the Li Ka Shing Knowledge Institute of St. Michael's Hospital (Toronto, Ontario, Canada), which is funded by the King Saud University (Riyadh, Saudi Arabia). The funder had no role in the conception or conduct of the project, the acquisition of data, or the decision to submit for publication. Dr. Straus is funded by a Tier 1 Canada Research Chair in Knowledge Translation and Dr. Tricco by a Canadian Institutes for Health Research/ Drug Safety and Effectiveness Network New Investigator Award in Knowledge Synthesis.

Previous presentation: Abstract presentation at the 117th American Academy of Otolaryngology-Head and Neck Surgery Annual Meeting \& OTO EXPO (AAOHNSF 2013), Vancouver, Canada [1]. 


\section{References}

1. Fnais N, Soobiah C, Idriss H, Dong B, Straus S, Tricco A. Diagnostic value of FNA-BRAF mutation analysis in papillary thyroid cancer: a systematic review and meta-analysis. Otolaryngol Head Neck Surg. 2013;149:P168-P169.

2.Hundahl SA, Fleming ID, Fremgen AM, Menck HR. A National Cancer Data Base report on 53,856 cases of thyroid carcinoma treated in the U.S., 19851995. Cancer. 1998;83:2638-2648.

3. Paterson IC, Greenlee R, Adams Jones D. Thyroid cancer in Wales 19851996: a cancer registry-based study. Clin Oncol. 1999;11:245-251.

4. Howlader N, Noone AM, Krapcho M, et al. SEER Cancer Statistics Review, 1975-2009 (Vintage 2009 Populations). eds Bethesda, MD: National Cancer Institute; April 2012. http://seer.cancer.gov/csr /1975_2009_pops09/. Accessed Sep 30, 2013.

5. Nucera C, Lawler J, Hodin R, Parangi S . The BRAFV600E mutation: what is it really orchestrating in thyroid cancer? Oncotarget. 2010;1:751-756.

6. Xing M, Tufano RP, Tufaro AP, et al. Detection of BRAF mutation on fine needle aspiration biopsy specimens: A new diagnostic tool for papillary thyroid cancer. J Clin Endocrinol Metab. 2004;89(6):2867-72.

7. Kim SW, Lee JI, Kim JW, et al. BRAFV600E mutation analysis in fine-needle aspiration cytology specimens for evaluation of thyroid nodule : a large series in a BRAF V600E-prevalent population . J Clin Endocrinol Metab. 2010; 95(8):3693-3700.

8. Namba H, Nakashima M, Hayashi T, et al. Clinical Implication of hot spot BRAF mutation, V599E, in papillary thyroid cancers. J Clin Endocrinol Metab. 2003;88:4393-4397. 
9. Xing M. BRAF mutation in thyroid cancer. Endoc Relat Cancer. 2005;12 :245-262.

10. Peyssonnaux C, Eychene A. The Raf/MEK/ERK pathway: new concepts of activation. Biol Cell. 2001;93:53-62.

11. Hilger RA, Scheulen ME, Strumberg D. The Ras-Raf-MEK-ERK pathway in the treatment of cancer. Onkologie. 2002;25:511-518.

12. Bentz BG, Miller BT, Holden JA, et al. B-RAF V600E mutational analysis of fine needle aspirates correlates with diagnosis of thyroid nodules. Otolaryngol Head Neck Surg. 2009;140(5):709-14.

13. Jemal A, Siegel R, Xu J, Ward E. Cancer Statistics , 2010 .Ca Cancer J Clin. 2010;60:277-300.

14. Nakayama H, Yoshida A, Nakamura Y, et al . Clinical Significance of BRAF (V600E) Mutation and Ki-67 Labeling Index in Papillary Thyroid Carcinomas. Anticancer Res. 2007;27:3645-3650.

15. Nikiforova MN, Kimura ET, Gandhi M, et al. BRAF mutations in thyroid tumors are restricted to papillary carcinomas and anaplastic or poorly differentiated carcinomas arising from papillary carcinomas. J Clin Endocrinol Metab. 2003;88(11):5399-404.

16. Riesco-Eizaguirre G, Gutiérrez-Martínez P, García-Cabezas MA, Nistal M, Santisteban P. The oncogene BRAF V600E is associated with a high risk of recurrence and less differentiated papillary thyroid carcinoma due to the impairment of $\mathrm{Na}+/ \mathrm{I}-$ targeting to the membrane. Endocr Relat Cancer. 2006;13(1):257-69.

17. American Thyroid Association (ATA) Guidelines Taskforce on Thyroid Nodules and Differentiated Thyroid Cancer, Cooper DS, Doherty GM, 
Haugen BR, et al. Revised American Thyroid Association management guidelines for patients with thyroid nodules and differentiated thyroid cancer. Thyroid. 2009;19:1167-1214.

18. Pacini F, Schlumberger M, Dralle H, et al. European consensus for the management of patients with differentiated thyroid carcinoma of the follicular epithelium. Eur J Endocrinol. 2006;154:787-803.

19. Cibas CS, Ali SZ, NCI Thyroid FNA State of the Science Conference. The Bethesda System For Reporting Thyroid Cytopathology. Am J Clin Pathol. 2009;132(5):658-665.

20. Chung KW, Yang SK, Lee GK, et al. Detection of BRAFV600E mutation on fine needle aspiration specimens of thyroid nodule refines cyto-pathology diagnosis, especially in BRAF600E mutation-prevalent area. Clin Endocrinol (Oxf). 2006;65(5):660-6.

21. Cohen Y, Rosenbaum E, Clark DP, et al. Mutational analysis of BRAF in fine needle aspiration biopsies of the thyroid: A potential application for the preoperative assessment of thyroid nodules. Clin Cancer Res. 2004;10(8):2761-5.

22. Chang HY, Lee H, Yoon SO, et al. BRAFV600Emutation analysis of liquidbased preparation-processed fine needle aspiration sample improves the diagnostic rate of papillary thyroid carcinoma. Hum Pathol. 2012;43(1):89-95.

23. Shamseer L, Moher D, Clarke M, et al. Preferred reporting items for systematic review and meta-analysis protocols (PRISMA-P) 2015: elaboration and explanation. BMJ. 2015;349:g7647.

24. Reitsma JB, Rutjes AWS, Whiting P, Vlassov VV, Leeflang MMG, Deeks JJ,. Chapter 9: Assessing methodological quality. In: Deeks JJ, Bossuyt PM, 
Gatsonis C (editors), Cochrane Handbook for Systematic Reviews of Diagnostic Test Accuracy Version 1.0.0. The Cochrane Collaboration, 2009.

25. Arends L, Hamza T, Van Houwelingen J, Heijenbrok-Kal M, Hunink M, Stijnen T. Bivariate Random Effects Meta-Analysis of ROC Curves. Med Decis Making. 2008;28(5):621-638.

26. Reitsma J, Glas A, Rutjes A, Scholten R, Bossuyt P, Zwinderman A. Bivariate analysis of sensitivity and specificity produces informative summary measures in diagnostic reviews. J Clin Epidemiol. 2005; 58:(10):982-990.

27. R Development Core Team (2011). R: A language and environment for statistical computing. R Foundation for Statistical Computing, Vienna, Austria. ISBN 3-900051-07-0, URL http://www.R-project.org/

28. Hayashida N, Namba H, Kumagai A, et al. A rapid and simple detection method for the BRAF(T1796A) mutation in fine-needle aspirated thyroid carcinoma cells. Thyroid. 2004;14(11):910-5.

29. Salvatore G, Giannini R, Faviana P, et al. Analysis of BRAF point mutation and RET/PTC rearrangement refines the fine-needle aspiration diagnosis of papillary thyroid carcinoma. J Clin Endocrinol Metab. 2004;89(10):5175-80.

30. Domingues R, Mendonca E, Sobrinho L, et al. Searching for RET/PTC rearrangements and BRAF V599E mutation in thyroid aspirates might contribute to establish a preoperative diagnosis of papillary thyroid carcinoma. Cytopathology. 2005;16(1):27-31.

31. Jin L, Sebo TJ, Nakamura N, et al. BRAF mutation analysis in fine needle aspiration (FNA) cytology of the thyroid. Diagn Mol Pathol. 2006;15(3):13643. 
32. Rowe LR, Bentz BG, Bentz JS. Detection of BRAF V600E activating mutation in papillary thyroid carcinoma using PCR with allele-specific fluorescent probe melting curve analysis. J Clin Pathol. 2007;60(11):1211-5.

33. Pizzolanti G, Russo L, Richiusa P, et al. Fine-needle aspiration molecular analysis for the diagnosis of papillary thyroid carcinoma through BRAF V600E mutation and RET/PTC rearrangement. Thyroid. 2007;17(11):1109-15.

34. Sapio MR, Guerra A, Posca D, et al. Combined analysis of galectin-3 and BRAFV600E improves the accuracy of fine-needle aspiration biopsy with cytological findings suspicious for papillary thyroid carcinoma. Endocr Relat Cancer. 2007 Dec;14(4):1089-97.

35. Sapio MR, Posca D, Raggioli A, et al. Detection of RET/PTC, TRK and BRAF mutations in preoperative diagnosis of thyroid nodules with indeterminate cytological findings. Clin Endocrinol (Oxf). 2007;66(5):678-83.

36. Kim SK, Kim D-, Han HS, et al. Pyrosequencing analysis for detection of a BRAFV600E mutation in an FNAB specimen of thyroid nodules. Diagn Mol Pathol. 2008;17(2):118-25.

37. Jo YS, Huang S, Kim YJ, et al. Diagnostic value of pyrosequencing for the BRAF V600E mutation in ultrasound-guided fine-needle aspiration biopsy samples of thyroid incidentalomas. Clin Endocrinol (Oxf). 2009;70(1):139-44.

38. Kim SK, Song K-, Lim SD, et al. Clinical and pathological features and the BRAFV600E mutation in patients with papillary thyroid carcinoma with and without concurrent hashimoto thyroiditis. Thyroid. 2009;19(2):137-41.

39. Kwak JY, Kim EK, Chung WY, et al. Association of BRAFV600E mutation with poor clinical prognostic factors and US features in korean patients with papillary thyroid microcarcinoma. Radiology. 2009;253(3):854-60. 
40. Marchetti I, Lessi F, Mazzanti CM, et al. A morpho-molecular diagnosis of papillary thyroid carcinoma: BRAF V600E detection as an important tool in preoperative evaluation of fine-needle aspirates. Thyroid. 2009;19(8):837-42.

41. Moon HJ, Kwak JY, Kim EK, et al. The role of BRAFV600E mutation and ultrasonography for the surgical management of a thyroid nodule suspicious for papillary thyroid carcinoma on cytology. Ann Sur Oncol. 2009;16(11):3125-31.

42. Xing M, Clark D, Guan H, et al. BRAF mutation testing of thyroid fine-needle aspiration biopsy specimens for preoperative risk stratification in papillary thyroid cancer. J Clin Oncol. 2009;27(18):2977-82.

43. Zatelli MC, Trasforini G, Leoni S, et al. BRAF V600E mutation analysis increases diagnostic accuracy for papillary thyroid carcinoma in fine-needle aspiration biopsies. Eur J Endocrinol. 2009;161(3):467-73.

44. Yip L, Nikiforova MN, Carty SE, et al. Optimizing surgical treatment of papillary thyroid carcinoma associated with BRAF mutation. Surgery. 2009;146(6):1215-23.

45. Nikiforov YE, Steward DL, Robinson-Smith TM, et al. Molecular testing for mutations in improving the fine-needle aspiration diagnosis of thyroid nodules. J Clin Endocrinol Metab. 2009;94(6):2092-8.

46. Cantara S, Capezzone M, Marchisotta S, et al. Impact of proto-oncogene mutation detection in cytological specimens from thyroid nodules improves the diagnostic accuracy of cytology. J Clin Endocrinol Metab. 2010;95(3):1365-9. 
47. Girlando S, Cuorvo LV, Bonzanini M, et al. High prevalence of B-RAF mutation in papillary carcinoma of the thyroid in north-east italy. Int J Surg Pathol. 2010;18(3):173-6.

48. Guo F, Hou P, Shi B. Detection of BRAF mutation on fine needle aspiration biopsy specimens: Diagnostic and clinical implications for papillary thyroid cancer. Acta Cytol. 2010;54(3):291-5.

49. Hwang J, Shin JH, Han B-, et al. Papillary thyroid carcinoma with BRAFV600E mutation: Sonographic prediction. Am J Roentgenol. 2010;194(5):W425-30.

50. Kwak JY, Kim E-, Kim J-, et al. Dual priming oligonucleotide-based multiplex PCR analysis for detection of BRAFV600E mutation in FNAB samples of thyroid nodules in BRAF V600E mutation-prevalent area. Head Neck. 2010;32(4):490-8.

51. Lee SJ, Lee MH, Kim DW, et al. Cross-regulation between oncogenic BRAF(V600E) kinase and the MST1 pathway in papillary thyroid carcinoma. PLoS ONE. 2011;6(1):e16180.

52. Lin KL, Wang OC, Zhang XH, Dai XX, Hu XQ, Qu JM. The BRAF mutation is predictive of aggressive clinicopathological characteristics in papillary thyroid microcarcinoma. Ann Surg Oncol. 2010;17(12):3294-300.

53. Musholt TJ, Fottner C, Weber MM, et al. Detection of papillary thyroid carcinoma by analysis of BRAF and RET/PTC1 mutations in fine-needle aspiration biopsies of thyroid nodules. World J Surg. 2010;34(11):2595-603.

54. Ohori NP, Nikiforova MN, Schoedel KE, et al. Contribution of molecular testing to thyroid fine-needle aspiration cytology of "follicular lesion of 
undetermined significance/atypia of undetermined significance". Cancer cytopathol. 2010;118(1):17-23.

55. Proietti A, Giannini R, Ugolini C, et al. BRAF status of follicular variant of papillary thyroid carcinoma and its relationship to its clinical and cytological features. Thyroid. 2010;20(11):1263-70.

56. Adeniran AJ, Hui P, Chhieng DC, et al. BRAF mutation in thyroid FNA specimens enhances predictability of malignancy in thyroid follicular lesion of undetermined significance. Acta Cytol. 2011;55(6):570-5.

57. Adeniran AJ, Theoharis C, Hui P, et al. Reflex BRAF testing in thyroid fineneedle aspiration biopsy with equivocal and positive interpretation: A prospective study. Thyroid. 2011;21(7):717-23.

58. Colanta A, Arcila M, Tafe L, et al. BRAF Mutation Analysis of Fine-Needle Aspiration Biopsies of Papillary Thyroid Carcinoma: Impact on Diagnosis and Prognosis. Acta Cytol. 2011;55(6):563-9.

59. Kim SK, Hwang TS, Yoo YB, et al. Surgical results of thyroid nodules according to a management guideline based on the BRAFV600E mutation status. J Clin Endocrinol Metab. 2011;96(3):658-64.

60. Moon HJ, Kim EK, Chung WY, et al. Diagnostic value of BRAF(V600E) mutation analysis of thyroid nodules according to ultrasonographic features and the time of aspiration. Ann Surg Oncol. 2011;18(3):792-9.

61. Pelizzo MR, Boschin IM, Barollo S, et al. BRAF analysis by fine needle aspiration biopsy of thyroid nodules improves preoperative identification of papillary thyroid carcinoma and represents a prognostic factor. A monoinstitutional experience. Clin Chem Lab Med. 2011;49(2):325-9. 
62. Kang G, Cho EY, Shin JH, Chung JH, Kim JW, Oh YL. Role of BRAFV600E mutation analysis and second cytologic review of fine-needle aspiration for evaluating thyroid nodule. Cancer Cytopathol. 2012;120(1):44-51.

63. Moon WJ, Choi N, Choi JW, Kim SK, Hwang TS. BRAF mutation analysis and sonography as adjuncts to fine-needle aspiration cytology of papillary thyroid carcinoma: Their relationships and roles. Am J Roentgenol. 2012;198(3):668-674.

64. Rossi M, Buratto M, Bruni S, et al. Role of ultrasonographic/clinical profile, cytology, and BRAF V600E mutation evaluation in thyroid nodule screening for malignancy: a prospective study. J Clin Endocrinol Metab. 2012;97(7):2354-61

65. Ohori NP, Singhal R, Nikiforova MN, et al. BRAF mutation detection in indeterminate thyroid cytology specimens: underlying cytologic, molecular, and pathologic characteristics of papillary thyroid carcinoma. Cancer Cytopathol. 2013;121(4):197-205.

66. Rossi ED, Martini M, Capodimonti S, et al. BRAF (V600E) mutation analysis on liquid-based cytology-processed aspiration biopsies predicts bilaterality and lymph node involvement in papillary thyroid microcarcinoma. Cancer Cytopathol. 2013;121(6):291-297.

67. Aragon Han P, Olson MT, Fazeli R, et al. The impact of molecular testing on the surgical management of patients with thyroid nodules. Ann Surg Oncol. 2014;21(6):1862-1869.

68. Hyeon J, Ahn S, Shin JH, Oh YL. The prediction of malignant risk in the category "atypia of undetermined significance/follicular lesion of undetermined significance" of the Bethesda System for Reporting Thyroid 
Cytopathology using subcategorization and BRAF mutation results. Cancer Cytopathol. 2014;122(5):368-376.

69. Poller DN, Glaysher S, Agrawal A, Caldera S, Kim D, Yiangou C. BRAF V600 co-testing in thyroid FNA cytology: Short-term experience in a large cancer centre in the UK. J Clin Pathol. 2014;67(8):684-689.

70. Jia Y, Yu Y, Li X, et al. Diagnostic value of B-RAF(V600E) in difficult-todiagnose thyroid nodules using fine-needle aspiration: systematic review and meta-analysis. Diagn Cytopathol. 2014;42(1):94-101.

71. Lee JH, Lee ES, Kim YS. Clinicopathologic significance of BRAF V600E mutation in papillary carcinomas of the thyroid: a meta-analysis. Cancer. 2007;110(1):38-46.

72. Xing M, Alzahrani AS, Carson KA, et al. Association between BRAF V600E mutation and mortality in patients with papillary thyroid cancer. JAMA. 2013;309(14):1493-501.

73. Howell GM, Nikiforova MN, Carty SE, et al. BRAF V600E mutation independently predicts central compartment lymph node metastasis in patients with papillary thyroid cancer. Ann Surg Oncol. 2013;20(1):47-52.

74. Joo JY, Park JY, Yoon YH, et al. Prediction of occult central lymph node metastasis in papillary thyroid carcinoma by preoperative BRAF analysis using fine-needle aspiration biopsy: a prospective study. J Clin Endocrinol Metab. 2012;97(11):3996-4003.

75. Xing M. BRAF mutation in papillary thyroid cancer: pathogenic role, molecular bases, and clinical implications. Endocr Rev. 2007;28(7):742-762. 
76. Chudova D, Wilde JI, Wang ET et al. Molecular classification of thyroid nodules using high- dimensionality genomic data. J Clin Endocrin Metab 2010;95(12):5296-304.

77. DeGroot LJ, Kaplan EL, McCormick M, Straus FH. Natural history, treatment, and course of papillary thyroid carcinoma. J Clin Endocrinol Metab. 1990;71(2):414-424.

78. Lijmer JG1, Mol BW, Heisterkamp S, et al. Empirical evidence of designrelated bias in studies of diagnostic tests. JAMA. 1999;282(11):1061-6. 
Figure 1: Study flow-chart

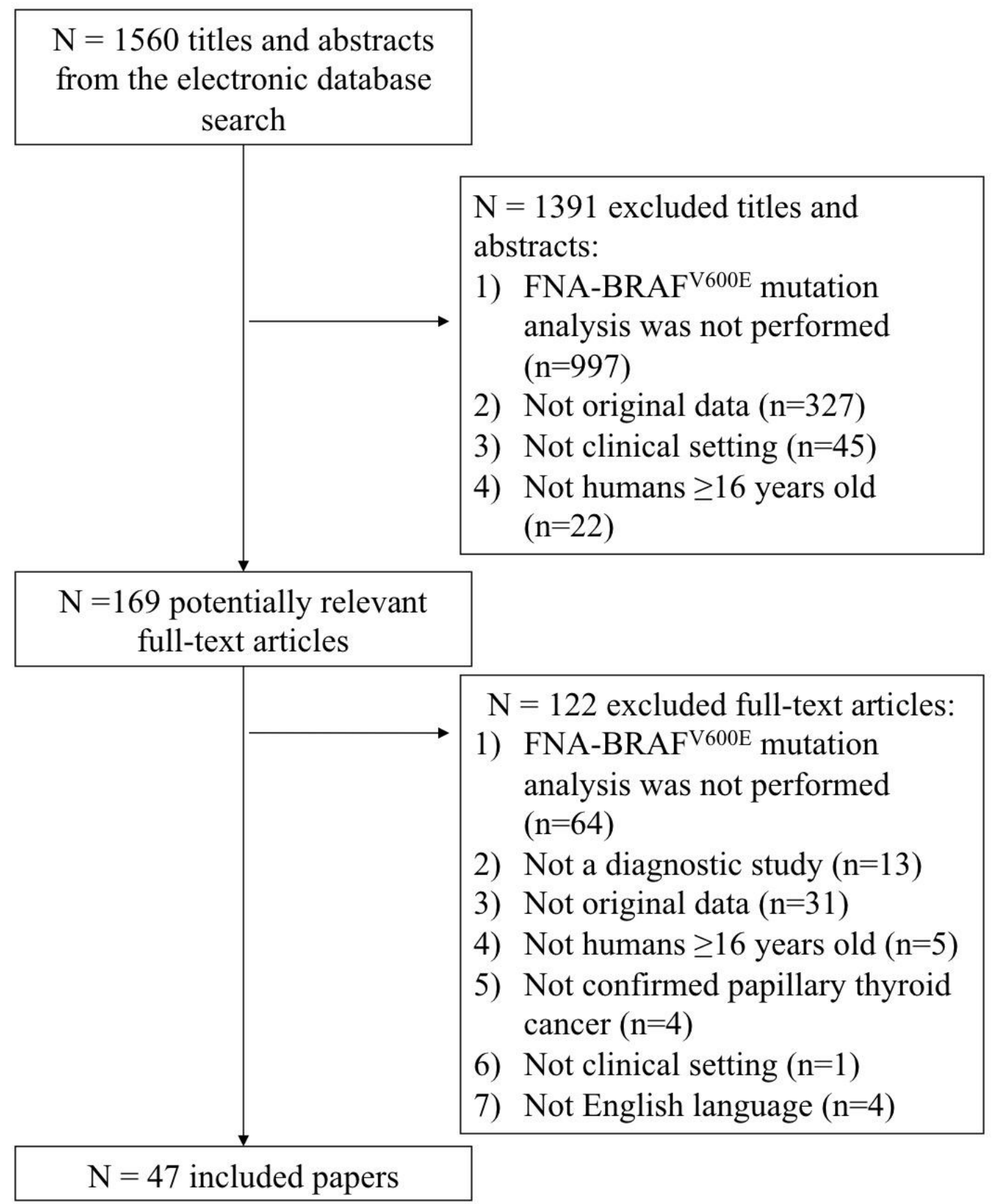

Figure 1: Flowchart of the literature search and study selection process in a 2012 systematic review of the literature on the diagnostic value of FNA-BRAF $F^{V 600 E}$ mutation analysis in papillary thyroid cancer. 
Figure 2: Summary measures for sensitivity of $B R A F^{V 600 E}$ mutation analysis in indeterminate group

\begin{tabular}{|c|c|c|c|c|c|c|}
\hline Author and Year & TP & $\mathrm{FN}$ & & & Weight & Sen $[95 \% \mathrm{Cl}]$ \\
\hline Cohen, 2004 & 5 & 24 & $\longmapsto$ & & $20.26 \%$ & $0.17[0.03,0.31]$ \\
\hline Xing, 2004 & 2 & 11 & $\longmapsto$ & & $18.31 \%$ & $0.15[0.00,0.35]$ \\
\hline Rowe, 2006 & 3 & 16 & $\longmapsto$ & & $19.41 \%$ & $0.16[0.00,0.32]$ \\
\hline Kim, 2008 & 13 & 5 & & $\longmapsto$ & $17.92 \%$ & $0.72[0.52,0.93]$ \\
\hline Cantara, 2010 & 2 & 5 & $\longmapsto$ & $\longrightarrow$ & $13.44 \%$ & $0.29[0.00,0.62]$ \\
\hline Pelizzo, 2011 & 2 & 3 & & & $10.66 \%$ & $0.40[0.00,0.83]$ \\
\hline \multirow[t]{4}{*}{ Pooled, $I^{2}=79.54 \%$} & & & & & $100 \%$ & $0.30[0.11,0.50]$ \\
\hline & & & $\Gamma$ & 1 & & \\
\hline & & & 0.00 & 0.50 & & \\
\hline & & & Sen & itivity (Sen) & & \\
\hline
\end{tabular}


Figure 3: Summary measures for sensitivity of $B R A F^{V 600 E}$ mutation analysis in suspicious for malignancy group

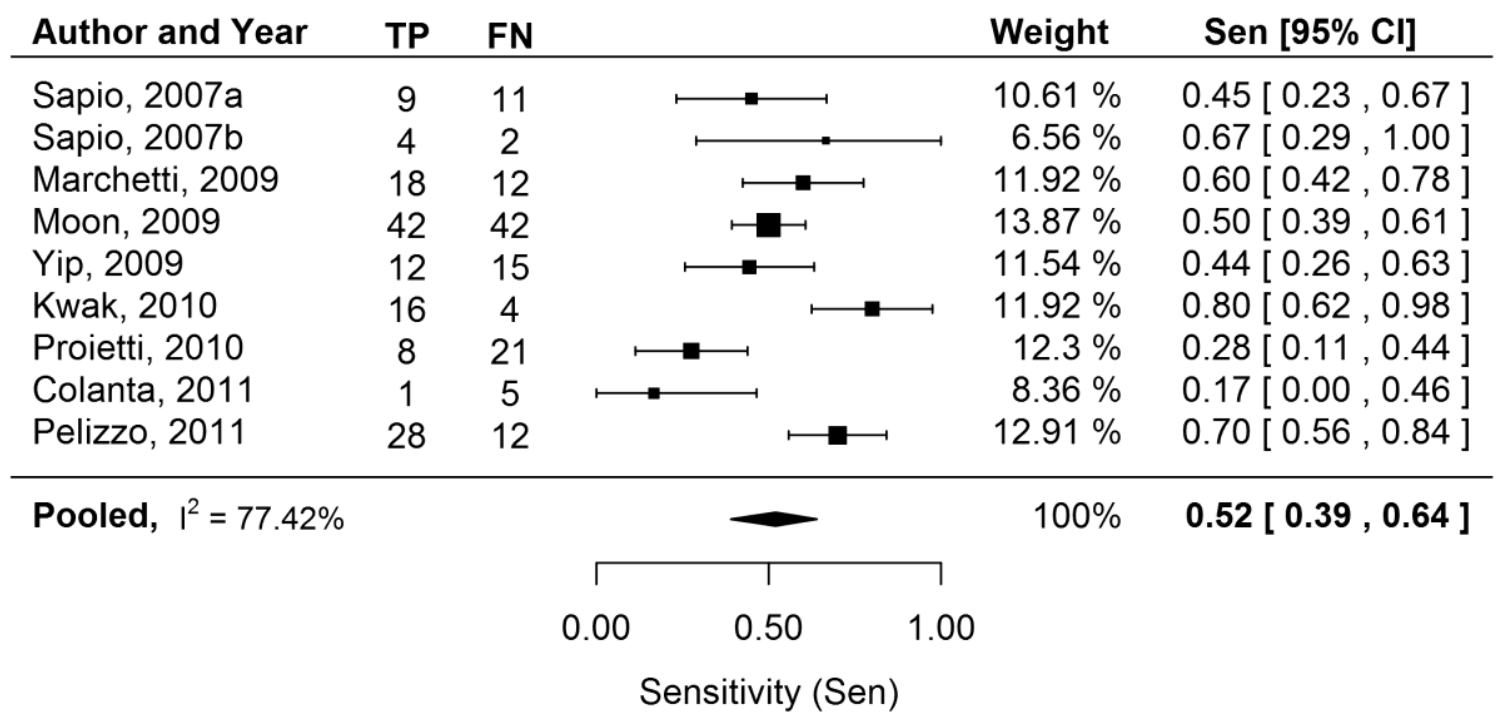


Figure 4: Summary measures for sensitivity of $B R A F^{V 600 E}$ mutation analysis in PTC group

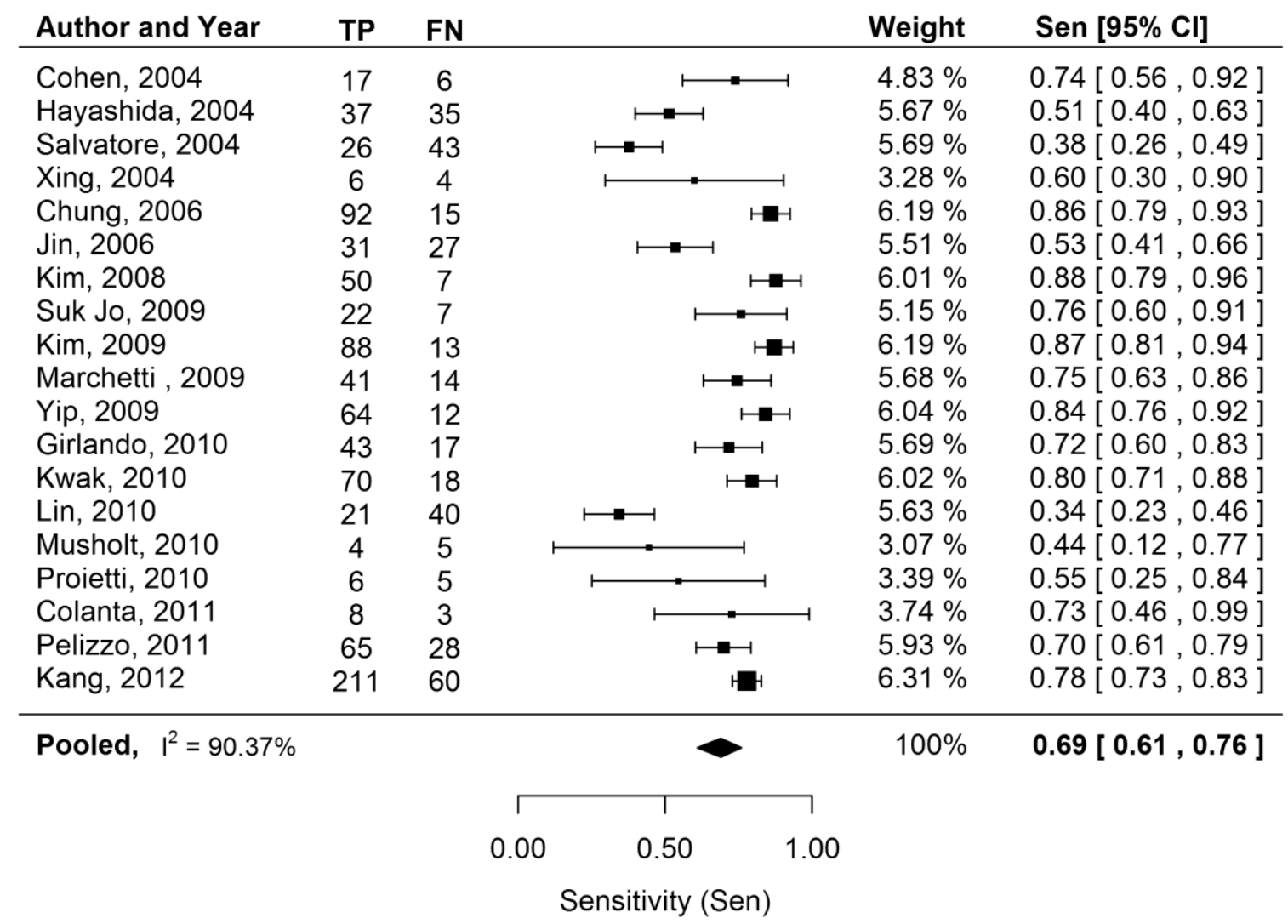


Figure 5: Summary measures for sensitivity and specificity of $B R A F^{V 600 E}$ mutation analysis in RET/PTC rearrangement

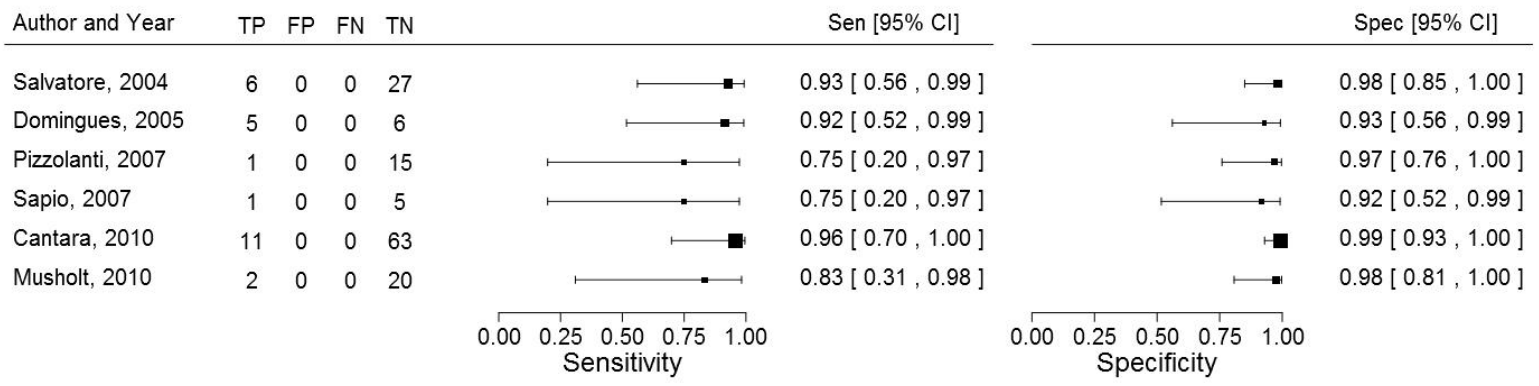

A. Coupled forest; for studies with one or more zero counts, 0.5 is added to the data.

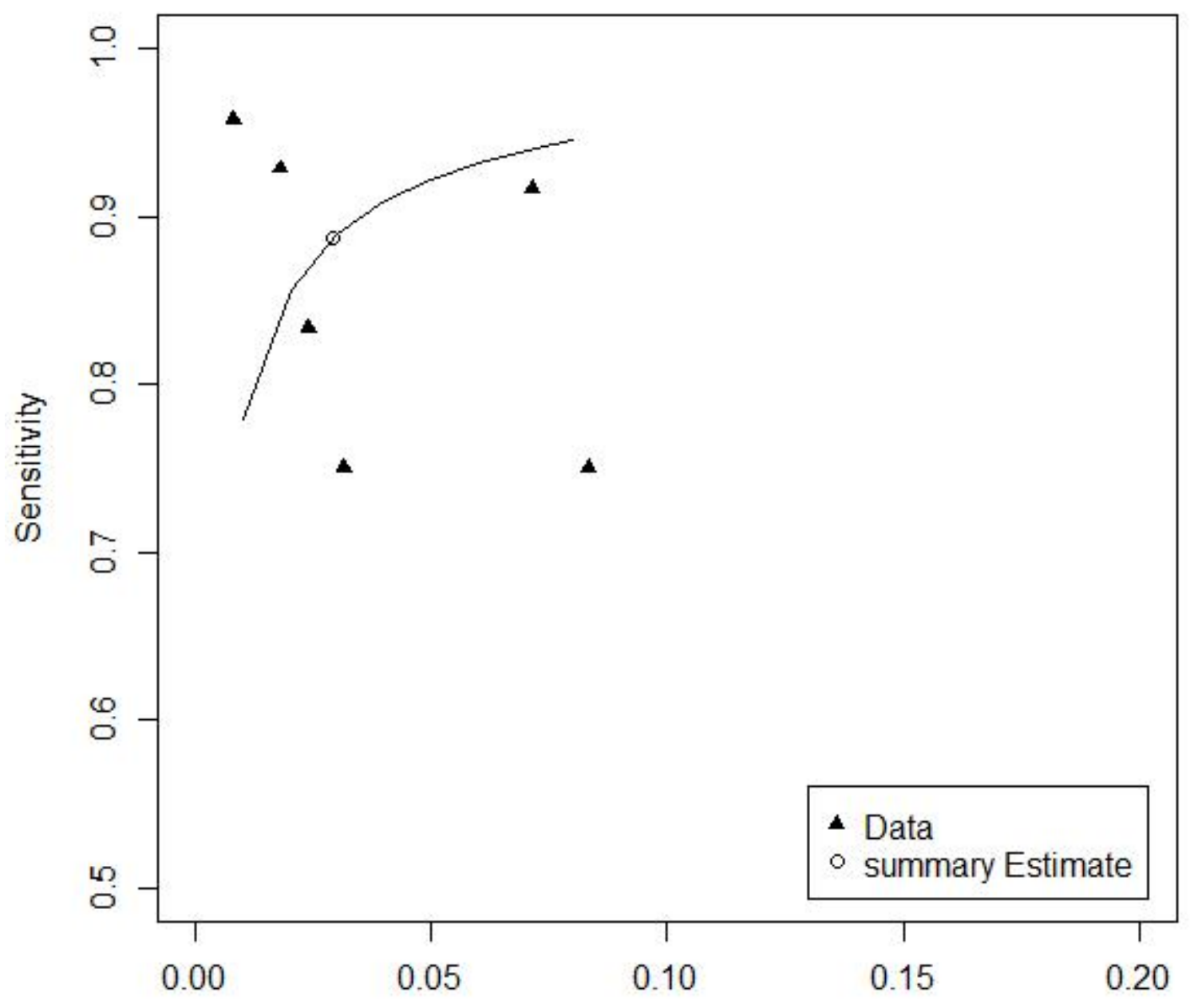

False Positive Rate

B. Summary ROC curve obtained from bivariate meta-analysis. 
Table 1: Study Characteristics

First Author

Country; Sitting

Study Design

Mean Age (SD)

Cohen et al 2004

1. [21]

USA; John Hopkins Medical Institution

Case control

NR

2. Hayashida et al 2004 [28] Japan; Nagasaki University Hospital in Japan

Cohort

53

Italy; Pathology Dept, University of Naples and

3. Salvatore et al 2004 [29]

University of Pisa

Case control

$42(16)$

4. $\quad$ Xing et al 2004 [6]

USA; John Hopkins Hospital (Baltimore, MD, USA)

Cohort

NR

5. Domingues et al 2005 [30]

Portugal; Instituto Portugues Oncologia

Cohort

NR

Chung et al 2006

Korea; Research Institute and Hospital National

6.

Cancer Center, Seoul National University Hospital

Cohort

46.4

7. Jin et al 2006 [31]

USA; Mayo Clinic, Rochester, MD, USA

Case control

52

8. $\quad$ Rowe et al 2006 [32]

USA; University of Utah Department of Surgery

Case control

NR

9. $\quad$ Pizzolanti et al 2007 [33]

Italy; Faculty of Medicine, University of Palermo

Cohort

$49.6(14.29)$

Italy; Azienda Osepdaliera Universitaria Federico II,

10. Sapio et al 2007 [34]

Naples; Ospedale Mauriziano, Turin

Case control

NR

Italy; Azienda Osepdaliera Universitaria Federico II,

11. Sapio et al 2007 [35]

Naples; Ospedale Mauriziano, Turin

Case control NR

Korea; Konkuk University Hospital

Case control

NR

13. Bentz et al 2009 [12]

USA; University of Utah Department of Surgery

Case control

$40.9(2.5)$

14.

Suk Jo et al 2009

Korea; Center for Endocrine Surgery, Chungham

National University Hospital

Cohort

NR

15. Kim et al 2009 [38]

Korea; Konkuk University Hospital

Case control

NR

16. Kwak et al 2009 [39]

Korea; Severance Hospital, Korea

Case control

45.2 
17. Marchetti et al 2009 [40]

Italy; Section of Cytopathology,Division of Surgical,

Molecular and Ultrastructural pathology

Case control

18. Moon et al 2009 [41]

Korea; Severance Hospital, Korea

Case control

45.7

19. Xing et al 2009 [42]

USA; John Hopkins Hospital (Baltimore, MD, USA) Case-series

(median) 44.5

20. Zatelli et al 2009

Italy; Section of Endocrinology, University of

Ferrara

Cohort

$50.7(0.7)$

21. Yip et al 2009 [44]

USA; University of Pittsburg Medical Center

Case control

NR

USA; University of Colorado Denver school of

22. Nikiforov et al 2009 [45]

Medicine, University of Cincinnati Medical Centre

Cohort

NR

Medicine, University of Cincinnati Medical Centre

23. Cantara et al 2010 [46] Italy; Department of Surgery, University of Sienna

Cohort

$51.2(13.8)$

24. Girlando et al 2010 [47]

Italy; North Italian population

Cohort

NR

25. Guo et al 2010 [48]

China; First Affiliated Hospital of Xi'an Jiaotong

University

Cohort

NR

Korea; Samsung Medical Centre, Sungkyurkwan

26. Hwang et al 2010 [49]

University school of Medicine

Cohort

47

Korea; Severance Hospital, Korea

Case control

45.5

27. Kwak et al 2010 [50]

Korea; Konkuk University Hospital

Cohort

$48.3(13.7)$

28. Lee et al 2010 [51]

China; First Affiliated Hospital of Wenzhou Medical

29. Lin et al 2010 [52]

College

Cohort

49.2

Germany; Mainz University (nuclear medicine,

30. Musholt et al 2010 [53]

endocrinology, pediatric endocrinology, endocrine

Cohort

NR

surgery), outpatient clinics in Mainz, and Augsburg

31. Ohori et al 2010 [54]

USA; University of Pittsburg Medical Center

Case control

NR

32. Proietti et al 2010 [55]

Italy; University Hospital of Pisa

Case control

44.7

33. Adeniran et al 2011 [56]

USA; NR (clinical)

Cohort

$52.6(15.6)$

USA; Department of Pathology, Diagnostic

34. Adeniran et al 2011 [57]

Radiology and Surgery, Yale University
49.4 (only those

Case control with BRAF

mutation) 
36. Kim et al 2011 [59]

37. Moon et al 2011 [60] Korea; Yonsei University College of Medicine

Case control

49.4

38. Pelizzo et al 2011 [61]

Italy; University Hospital of Padova

Cohort

47.8

Chang et al 2012

39. [22]

\section{Korea; Department of Pathology, Samsung Medical}

40. Kang et al 2012 [62]

\section{Center}

50

41. Moon et al 2012 [63] Korea; Konkuk University Medical Center

Case control

Italy; University of Ferrara, and Azienda

42. Rossi et al 2012 [64]

Ospedaliero Universitaria di Ferrara

Cohort

USA; University of Pittsburgh Medical Center-

43. Ohori et al 2013 [65]

Presbyterian

Case control

Age range (33.8

- 47.9)

Italy; Agostino Gemelli School of Medicine,

44. Rossi et al 2013 [66]

Catholic University of the Sacred Heart

Cohort

47 (median)

USA: The Johns Hopkins University School of

45.

Aragon et al 2014

Medicine

Case control

$46.7(13.5)$

[67]

Korea; Department of Pathology, Samsung Medical

46.

Hyeon et al 2014

Center

Case control

49.7

[68]

UK; Queen Alexandra Hospital

Case control

NR

47. Poller et al 2014 [69]

UK; Queen Alexandra Hospital

Abbreviation: NR, Not reported. 
Table 2: Patient Characteristics

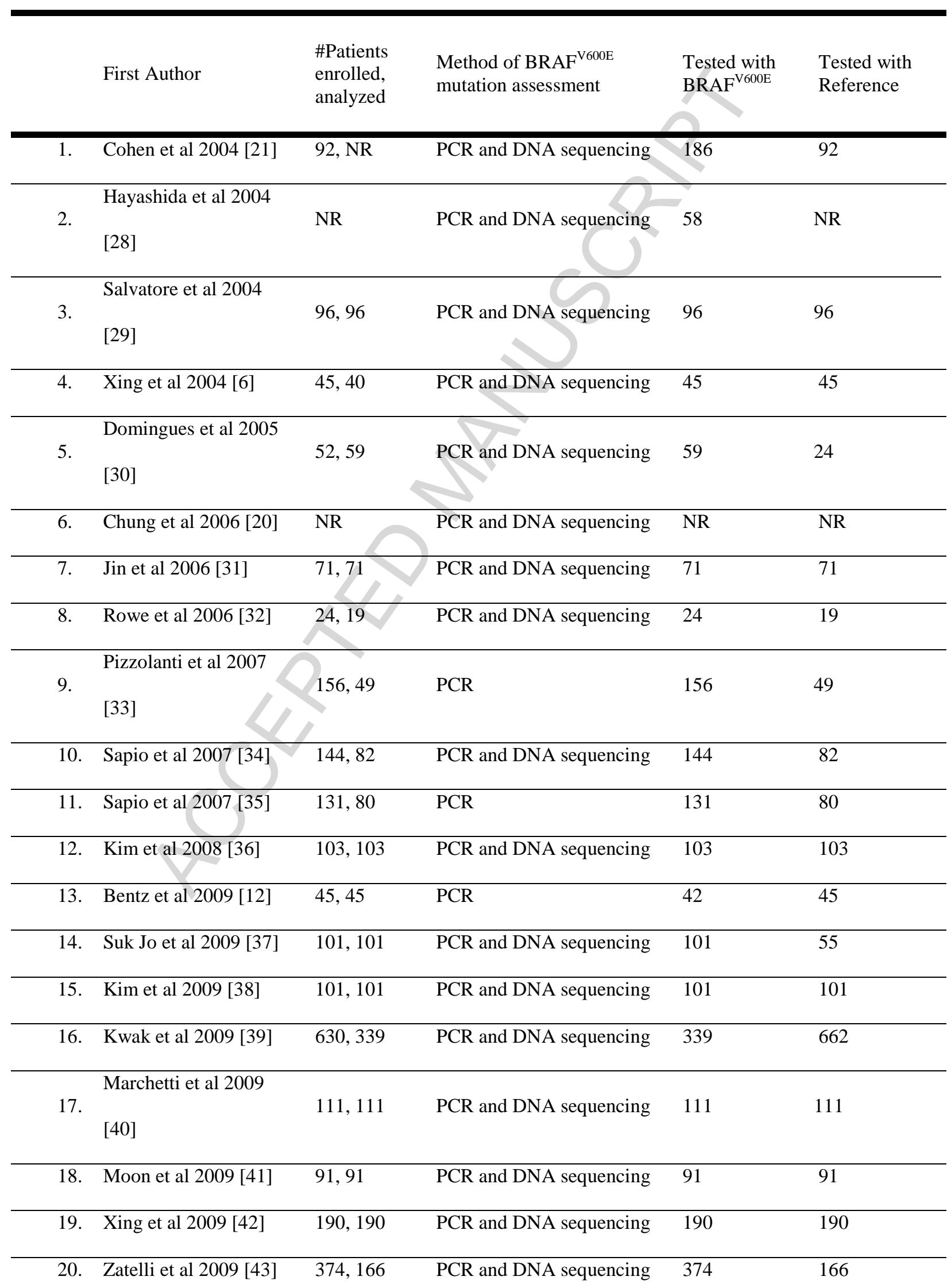


21. Yip et al 2009 [44] 332, 206 $\quad$ PCR 332

\begin{tabular}{|c|c|c|c|c|c|}
\hline 22. & $\begin{array}{l}\text { Nikiforov et al } 2009 \\
{[45]}\end{array}$ & 328,69 & PCR and DNA sequencing & 328 & 69 \\
\hline 23. & Cantara et al 2010 [46] & 174,174 & PCR & 235 & 174 \\
\hline 24. & $\begin{array}{l}\text { Girlando et al } 2010 \\
\text { [47] }\end{array}$ & NR & PCR and DNA sequenci & 91 & 64 \\
\hline 25. & Guo et al 2010 [48] & 10,10 & NR & 10 & 10 \\
\hline 26. & Hwang et al 2010 [49] & 135,135 & PCR and DNA sequencing & 135 & 135 \\
\hline 27. & Kwak et al 2010 [50] & 130,118 & PCR and DNA sequencing & 130 & 130 \\
\hline 28. & Lee et al 2010 [51] & 85,52 & $\begin{array}{l}\text { DPO system, and DNA } \\
\text { sequencing }\end{array}$ & 85 & 85 \\
\hline 29. & Lin et al 2010 [52] & 61,20 & PCR & 61 & 61 \\
\hline 30. & Musholt et al 2010 [53] & 290,83 & PCR and DNA sequencing & 290 & 93 \\
\hline 31. & Ohori et al 2010 [54] & NR & PCR & NR & NR \\
\hline 32. & Proietti et al 2010 [55] & 187,87 & PCR and DNA sequencing & 187 & 187 \\
\hline 33. & $\begin{array}{l}\text { Adeniran et al } 2011 \\
{[56]}\end{array}$ & 146 & PCR & 146 & 68 \\
\hline 34. & $\begin{array}{l}\text { Adeniran et } \\
{[57]}\end{array}$ & 81,81 & PCR and DNA sequencing & 84 & 84 \\
\hline 35. & Colanta et al 2011 [58] & NR, 52 & PCR and DNA sequencing & 52 & 52 \\
\hline 36. & Kim et al 2011 [59] & 865,194 & PCR & 849 & NR \\
\hline 37. & Moon et al 2011 [60] & 295,102 & PCR & 295 & 102 \\
\hline 38. & Pelizzo et al 2011 [61] & 270,270 & PCR and DNA sequencing & 270 & 270 \\
\hline
\end{tabular}

39. Chang et al 2012 [22] $\quad$ 191, $191 \quad$ PCR and DNA sequencing $\quad 191 \quad 191$

40. Kang et al 2012 [62] $\quad 1060,1060 \quad$ PCR and DNA sequencing $1060 \quad 313$ 
41. Moon et al 2012 [63] 524,553 PCR and DNA sequencing 553

\begin{tabular}{rlllll}
\hline 42. & Rossi et al 2012 [64] & 1856, 1856 & PCR and DNA sequencing & 1856 & 325 \\
\hline 43. & Ohori et al 2013 [65] & 131,131 & PCR and DNA sequencing & 131 & 119 \\
\hline 44. & Rossi et al 2013 [66] & 230; NR & PCR and DNA sequencing & 73 & 50 \\
\hline 45. & Aragon et al 2014 [67] & $87 ; 87$ & NR & 29 & 29 \\
\hline 46. & Hyeon et al 2014 [68] & NR; 6402 & PCR and DNA sequencing & Unclear & Unclear \\
\hline 47. & Poller et al 2014 [69] & NR, 207 & PCR and DNA sequencing & 29 & 207
\end{tabular}

Abbreviation: NR, Not reported. 


\section{Highlights}

- FNA with cytological analysis is an initial step in diagnosing thyroid nodules that are suspicious for cancer.

- New genetic markers including $B R A F^{V 600 E}$ gene mutation analysis are available that may increase the accuracy of FNA for diagnosing thyroid cancer.

- The results of our study indicate a low sensitivity of $B R A F^{V 600 E}$ mutational analysis in diagnosing PTC.

- The feasibility of this test as a single molecular tool is not well established, which indicates the need for well-designed prospective clinical studies. 